\title{
Is white matter affected in adolescents with anorexia nervosa? A study using magnetization transfer imaging
}

\author{
Sinem Akgül ${ }^{1}$, Ayşegül Öz $z^{2}$, Kader Karl1-Oğuz ${ }^{2}$, Nuray Kanbur ${ }^{1}$, Orhan Derman ${ }^{1}$ \\ ${ }^{1}$ Division of Adolescent Medicine, Department of Pediatrics, ${ }^{2}$ Department of Radiology, Hacettepe University Faculty of \\ Medicine, Ankara, Turkey. Email:sinemhusnu@gmail.com \\ Received: 31 May 2016, Revised: 22 July 2016, Accepted: 29 July 2016
}

SUMMARY: Akgül S, Öz A, Karlı-Oğuz K, Kanbur N, Derman O. Is white matter affected in adolescents with anorexia nervosa? A study using magnetization transfer imaging. Turk J Pediatr 2016; 58: 282-286.

The aim of the study was to assess potential changes in structural integrity of the brain in adolescent patients with Anorexia Nervosa (AN) using the magnetization transfer imaging technique and re-evaluating after nutritional rehabilitation was accomplished.

Magnetization transfer ratio (MTR) was used for the detection and quantification of histological changes to the white matter of 9 adolescents diagnosed with AN at diagnosis and after weight gain. Using the MTR technique 6 regions were measured: the pons, left cerebellar hemisphere, amygdala, thalamus, corona radiata and white matter adjacent to the left dorsolateral prefrontal cortex. Scans from the patients with AN were also compared with scans from an age-matched subset of 9 healthy controls.

Compared with control subjects, the MTR of participants with AN was no different either at the early stages of diagnosis $(p=0.62)$ or after weight recovery $(p=0.81)$. Similarly, comparison of MTR between patients with AN at diagnoses compared with MTR after weight recovery yielded no statistically significant difference $(\mathrm{P}=0.33)$. We conclude that MTR was not able to detect any neuropathological alterations in adolescent patients with AN at either diagnosis or after nutritional recovery and the achievement of weight gain.

Key words: adolescent, anorexia nervosa, magnetization transfer imaging, magnetization transfer ratio.

Due to the advances in neuroimaging techniques both structural and functional brain changes have been detected within patients with anorexia nervosa $(\mathrm{AN})^{1}$. Changes such as dilation of the cerebral ventricles and sulci, increase in cerebral spinal fluid (CSF) and loss of brain parenchyma are now well known ${ }^{2}$.Although the main cerebral changes seem to be reversible to some extent after adequate weight restoration, ${ }^{3}$ studies have shown that structural brain changes such as gray matter volume deficits and elevated cerebrospinal fluid volumes may persist ${ }^{4}$. The exact pathogenesis of the damage remains unclear but research suggests that neuronal damage occurs secondary to chronic starvation and the regeneration of myelin accounts for the general reversibility ${ }^{1}$.

Although conventional magnetic resonance imaging (MRI) techniques are useful for demonstrating structural brain changes in patients with AN, these techniques are not optimally suited to reflect changes in the histological composition of the brain. In contrast, magnetization transfer imaging (MTI) permits a more accurate detection and quantification of histological changes and also detects structural changes that may not be visible with standard MRI techniques such as changes in myelin and cell membranes in white matter ${ }^{5}$. This technique is based on the exchange of magnetization between a pool of water protons and a pool of macromolecular (eg, myelin) bound protons in biological tissues. The exchange of magnetization is usually represented as the magnetization transfer ratio (MTR), where a decrease in ratio shows structural abnormality and/or damage ${ }^{6}$. 
The technique has frequently been used to evaluate the changes of normal appearing white matter in patients with multiple sclerosis and demonstrated occult white matter abnormalities. In MS patients MTR reduction is correlated with demyelination and axonal loss ${ }^{7}$. Despite the lack of specificity in its findings, the technique, has long been used in determination of tissue damage especially in conditions with no or minimal observable radiologic abnormality and with clinic-radiologic discrepancy. These conditions include traumatic brain injury, neurodegenerative diseases especially at the presymptomatic stage and monitoring of treatment related changes on brain parenchyma. ${ }^{8-10}$

In general, the majority of the studies on AN have reported brain volume deficits and increased cerebrospinal fluid. 11,12 To our knowledge, no previous study has evaluated cerebral tissue abnormalities occurring in patients with anorexia neither during the disorder nor following treatment using MTI.

In this study we investigated potential changes in structural integrity of the brain by comparing the MTR changes seen with those patients that nutritional rehabilitation has been accomplished comparing with a control group.

\section{Material and Methods}

\section{Subjects}

This prospective study took place at Hacettepe University, Department of Pediatrics, Division of Adolescent Medicine and the Department of Radiology. The study population consisted of 9 ( 1 male, 8 female) adolescents diagnosed with AN according to the fourth edition of Diagnostic and Statistical Manual of Mental Disorders (DSM-IV). Exclusion criteria were medical instability, chronic medical illness, chronic drug use including substance use/abuse, head trauma, learning disability, intellectual impairment, or metallic implants. Age, sex, anthropometric measures and duration of illness were recorded.

Weight and height were measured in all of the patients and their BMI was calculated (weight [kilograms]/height [meters squared]). Tanner staging was performed for every patient. Information regarding menstrual status was obtained. The initial scan was obtained when patients were medically stable. For 2 patients this was at diagnosis. For the remaining 7 patients this was in the early stage of treatment. The second scan was obtained after nutritional rehabilitation and weight recovery. The target weight used for recovery was calculated according to median weightfor-height adjusted for age also considering the patients' growth charts for the male and with the additional consideration of weight at regaining spontaneous menses for females.

The control subjects were 9 ( 1 male, 8 female) healthy adolescents of the same age recruited from the adolescent outpatient clinic. Indication for a cranial MRI was for work-up of headaches. All control subjects were of normal weight, had no chronic or psychiatric illness, no history of head trauma and had a normal neurological examination; a detailed history was obtained to rule out eating-disordered behavior.

Written informed consent was obtained from the parents and adolescents for both the patient and control groups. The Research Ethics Board of Hacettepe University approved this study.

\section{Image evaluation and analysis}

MR imaging was performed on a $1.5 \mathrm{~T}$ unit system (Intera Achieva, Philips, Netherlands) using a standard head coil. Initially $\mathrm{T} 2$ weighted (W) fast spin-echo (SE) (repetition time (TR)/ echo time (TE); 4000/100ms, matrix: 448x88) and T1W SE (TR/TE; 550/15 ms) axial acquisition were performed. Two radiologists evaluated these images for image quality and exclusion of an incidental lesion. Then, 3D T1-W fast field echo MTI sequence was obtained (repetition time (TR):37 ms, echo time (TE):4.4 ms, flip angle: $8^{\circ}$, matrix of $256 \times 256$ and a FOV: $240 \mathrm{~mm}$ ). Twenty-four contiguous $4 \mathrm{~mm}$ axial slices without gap, covering the whole brain, were acquired both with and without presaturation. The saturating MT pulse was 100 $\mathrm{Hz}$ off resonance. Saturated and unsaturated images were interleaved resulting in inherently registered saturated and unsaturated data sets.

Following data acquisition, the radiologists who were blinded to patients and healthy control subjects performed image postprocessing. On a workstation provided by the manufacturer (Viewforum Version 5, Philips, Netherlands) using a local thresholding segmentation technique the extracerebral tissue was removed 
and MTR maps derived pixel by pixel were obtained.

From the MTR maps, manual outlining of region-of-interests (ROI) of $12-15 \mathrm{~mm}^{2}$ in size were outlined from 6 regions: the pons, left cerebellar hemisphere, amygdala, thalamus, corona radiata and white matter adjacent to the left dorsolateral prefrontal cortex. These ROIs were selected either because of their compact myelin content or their potential relation with eating behavior and cognition.

\section{Statistical analysis}

Data with normal distribution were expressed as mean \pm standard deviation. MTR for the first and second scan from 6 regions (the pons, left cerebellar hemisphere, amygdala, thalamus, corona radiata and white matter adjacent to the left dorsolateral prefrontal cortex) was compared using paired samples T-test. The first and second scan was additionally compared with the control group using the independent samples T-test. $\mathrm{P} \leq .05$ was considered significant. All statistical analyses were performed using SPSS ${ }^{\circledR} 21$ statistical software.

\section{Results}

Group means and standard deviations for demographic and weight variables for patients with AN at first and second scan and for the control group are presented in Table I. All female patients in the study were amenorrheic when first scan was performed.

The first conventional MRI scan revealed widening of the cerebral sulci for 7 patients with no other gross abnormalities. In the second scan taken after weight recovery this finding had disappeared in 4 patients but remained in 3 .

The average length of recovery was 14.1 months \pm 4.1 (min: 9 months- max: 24 months).

Compared with control subjects, the MTR of participants with AN was no different either at diagnosis $(p=0.62)$ or after weight recovery $(p=0.81)$ (Table II). Similarly comparison of MTR between patients with AN at the early stages of diagnoses compared with MTR after weight recovery yielded no statistically significant difference $(\mathrm{P}=0.33)$ (Table II).

\section{Discussion}

Adolescence is the intellectual, physical, hormonal and social developmental phase full

Table I. Characteristics of Patients and Control Group

\begin{tabular}{lccc}
\hline & \multicolumn{2}{c}{ Anorexia group } & Control group \\
\cline { 2 - 4 } & First MRI & $\begin{array}{c}\text { Second } \\
\text { MRI }\end{array}$ & $16.1 \pm 2.3$ \\
\hline Age at MRI (yrs) & $15.8 \pm 2.5$ & $17.1 \pm 2.8$ & $12.3-21$ \\
BMI at MRI $\left(\mathrm{kg} / \mathrm{m}^{2}\right)$ & $(13-21)$ & $14-22)$ & $22.6 \pm 2.3$ \\
Illness duration (months) & $16.3 \pm 1.9$ & $19.2 \pm 1.3$ & NA \\
\hline
\end{tabular}

All figures shown are mean \pm SD followed by range.

BMI: Body mass index; MRI: Magnetic resonance imaging; NA:Not applicable. ${ }^{*} \mathrm{p}<0.05$

Table II. Comparison of MTR (\%)

\begin{tabular}{|c|c|c|c|c|c|c|}
\hline & DLPFC & $\begin{array}{l}\text { Corona } \\
\text { Radiata }\end{array}$ & Thalamus & Amygdala & $\mathrm{LCH}$ & Pons \\
\hline $\begin{array}{l}\text { MTR of first } \\
\text { scan in AN } \\
\text { patients }\end{array}$ & $57.51 \pm 2.7$ & $58.52 \pm 3.2$ & $\begin{array}{c}56.5 \\
\pm 3.96\end{array}$ & $\begin{array}{l}55.15 \\
\pm 2.64\end{array}$ & $49.68 \pm 6.75$ & $60.16 \pm 2.94$ \\
\hline $\begin{array}{l}\text { MTR of second } \\
\text { scan in AN } \\
\text { patients }\end{array}$ & $\begin{array}{c}57.12 \pm \\
\quad 2.6\end{array}$ & $57.57 \pm 3.6$ & $\begin{array}{l}55.28 \\
\pm 4.09\end{array}$ & $53.36 \pm 2.8$ & $53.01 \pm 3.8$ & $58.45 \pm 2.87$ \\
\hline $\begin{array}{l}\text { MTR of control } \\
\text { group }\end{array}$ & $56.7 \pm 3.5$ & $57.87 \pm 4.7$ & $56 \pm 3.89$ & $54.91 \pm 3.3$ & $56.7 \pm 3.05$ & $60.18 \pm 2.67$ \\
\hline
\end{tabular}

All figures shown are mean, \pm SD

DLPFC: dorsolateral prefrontal cortex. LCH: left cerebellar hemisphere, MTR: Magnetization transfer ratio 
of changes and transformations. The pubertal transition to adulthood involves both gonadal and behavioral maturation. MRI studies have discovered that myelinogenesis, continues from childhood and the brain's regionspecific neurocircuitry remains structurally and functionally vulnerable to impulsive sex, food, and sleep habits. ${ }^{13}$ The maturation of the adolescent brain is also influenced by heredity, environment, and sex hormones, which play a crucial role in myelination. ${ }^{14}$ Changes in myelin can be detected more accurately by MTI than standard MRI techniques. ${ }^{5}$

Schwartz et al. ${ }^{15}$ investigated the cross-sectional relationship between visceral fat, total body fat and MTR in the brains of typically developing adolescents, in a community-based sample of 970 adolescents (12-18 years old, 466 males). A magnetization transfer (MT) sequence was used to obtain MTR in gray matter and white matter. They found that both larger volumes of visceral fat and more total body fat were independently associated with higher MTR in both gray and white matter. These relationships were found to be independent of a number of potential confounders, including age, sex, puberty stage and height. This study suggests that both visceral fat and fat deposited elsewhere in the body are associated independently with structural properties of the adolescent brain. The authors speculated that these relationships suggest the presence of adiposity-related variations in phospholipid composition of brain lipids in adolescents. ${ }^{15}$

A systematic review of the literature reports that during AN adolescent females lose more central body fat, while adult females more peripheral fat. Partial weight restoration leads to greater fat mass deposition in the trunk region than other body regions in adolescent females. The abnormal central fat distribution seems to normalize after long-term maintenance of complete weight restoration, indicating that preferential central distribution of body fat is a transitory phenomenon. However, a discrepancy in the findings has been noted, especially between adolescents and adults; besides age and gender, these appear to be related to differences in the methodology and time of body composition assessments. ${ }^{16}$ Yet it is unclear when and how the brain lipids are affected during the disease period and this is more complex in adolescents with AN since this is already a period of brain maturation as explained above.

Although many different hypotheses have been discussed in the literature concerning the cause of structural brain changes in patients with AN such as; dehydration and starvation leading to a loss of intracellular protein thus causing alterations in intracellular oncotic pressure, the exact causal mechanism is still open for debate. Another important argument that remains is whether these changes are reversible with weight gain. Some MRI studies have shown certain changes while underweight, such as enlarged lateral ventricles then normalized after weight recovery. A study by Golden et al. ${ }^{17}$ evaluated the difference in total ventricular volume between AN patients at diagnosis and a follow-up after nutritional rehabilitation using quantitative MRI. They found that follow-up ventricular volume decreased from admission returning to a normal range after sufficient nutritional rehabilitation. Whereas other authors would argue that some changes may be irreversible. A study by Katzman et al. ${ }^{4}$ evaluated patients after weight gain and showed that gray matter volume deficits persisted in patients even after weight recovery, suggesting an irreversible element of structural brain changes in AN patients. ${ }^{4}$

To date no previous study has evaluated cerebral tissue abnormalities occurring in adolescents with AN neither during the disorder nor following treatment using MTI which was assumed that may accurately detect adiposityrelated variations in phospholipid composition of brain lipids in adolescents. The results of our study suggest the MTR was not able to detect any alteration in AN patients at diagnosis. One could expect alterations in the cerebral tissue related with cognitive status such as limbic structures and dorsolateral prefrontal cortex, areas with condensed white matter such as corona radiata, pons and thalamus both during the disease and following treatment reflecting recovery in cerebral macromolecular tissue structure. However our study group failed to show any significant imaging differences between the AN and control group. A recent study also aimed to evaluate the impact of AN at diagnosis on white matter microstructure during adolescence using diffusion MRI. 
Contrary to our findings in this study they demonstrate that disturbances in white matter were present and that these changes were likely to reflect alterations in myelin content. ${ }^{18}$

An additional, aim of this study was to evaluate the reversibility of structural changes after weight rehabilitation but in this study the initial MTR was not statistically different from the scan after nutritional rehabilitation and weight recovery. A similar study in women with restricting-type AN also aimed to determine whether deficits in white matter microstructure are present, using diffusion tensor imaging, after long term recovery and comparing with healthy controls. They too found no significant alteration in recovered AN patients. ${ }^{19}$

We did not look at gray matter loss in this study; rather we focused on the magnetization transfer capacity of subcortical tissue. Tissue microstructural changes may not have occurred in our sample size as demonstrated by unaffected magnetization transfer between proton pools in the cerebral tissue.

Several limitations, which we are well aware of, exist for our study. Primarily the sample size was very small. Admittedly, our results might have been affected by the relatively low numbers of patients studied and so these results should be confirmed by a large scale. Additionally the duration of illness before diagnosis was different for each patient, which may also affect the results.

Further studies regarding adiposity-related variations in phospholipid composition of brain lipids focusing on adolescents are also essential especially for patients with AN to understand the reversibility of the functional impairment.

\section{REFERENCES}

1. Jáuregui-Lobera I. Neuroimaging in eating disorders. Neuropsychiatr Dis Treat 2011; 7: 577-584.

2. Chui HT, Christensen BK, Zipursky RB, et al. Cognitive function and brain structure in females with a history of adolescent-onset anorexia nervosa. Pediatrics 2008; 122: $426-437$

3. King JA, Geisler D, Ritschel F, et al. Global cortical thinning in acute anorexia nervosa normalizes following long-term weight restoration. Biol Psychiatry 2015; 77: 624-632.
4. Katzman DK, Zipursky RB, Lambe EK, Mikulis DJ. A longitudinal magnetic resonance imaging study of brain changes in adolescents with anorexia nervosa. Arch Pediatr Adolesc Med 1997; 151: 793-797.

5. Grossman RI, Gomori JM, Ramer KN, et al. Magnetization transfer: theory and clinical applications in neuroradiology. Radiographics 1994; 14: 279-290.

6. Van Buchem M, Tofts PS. Magnetization transfer imaging. Neuroimaging Clin N Am 2000; 10: 771-788.

7. Filippi M, Campi A, Dousset V, et al. A magnetization transfer imaging study of normal-appearing white matter in multiple sclerosis. Neurology 1995; 45: 478-482.

8. Van der Voorn JP, Pouwels PJ, Salomons GS, Barkhof F, van der Knaap MS. Unraveling pathology in juvenile Alexander disease: serial quantitative MR imaging and spectroscopy of white matter. Neuroradiology 2009; 51: 669-675.

9. Bagley LJ, McGowan JC, Grossman RI, et al. Magnetization transfer imaging of traumatic brain injury. J Magn Reson Imaging 2000; 11: 1-8.

10. Jurgens CK, Bos R, Luyendijk J, et al. Magnetization transfer imaging in 'premanifest'Huntington's disease. J Neurol 2010; 257: 426-432.

11. Ehrlich S, Burghardt R, Weiss D, et al. Glial and neuronal damage markers in patients with anorexia nervosa. J Neural Transm 2008; 115: 921-927.

12. Kraeft JJ, Uppot RN, Heffess AM. Imaging findings in eating disorders. AJR Am J Roentgenol 2013; 200: 328-335.

13. Giedd JN, Blumenthal J, Jeffries NO, et al. Brain development during childhood and adolescence: a longitudinal MRI study. Nat Neurosci 1999; 2: 861863.

14. Arain $M$, Haque $M$, Johal L, et al. Maturation of the adolescent brain. Neuropsychiatr Dis Treat 2013; 9: 449-461.

15. Schwartz DH, Dickie E, Pangelinan MM, et al. Adiposity is associated with structural properties of the adolescent brain. Neuro Image 2014; 103: 192-201.

16. El Ghoch M, Calugi S, Lamburghini S, et al. Anorexia nervosa and body fat distribution: a systematic review. Nutrients 2014; 6: 3895-3912.

17. Golden NH, Ashtari M, Kohn MR, et al. Reversibility of cerebral ventricular enlargement in anorexia nervosa, demonstrated by quantitative magnetic resonance imaging. J Pediatr 1996; 128: 296-301.

18. Travis KE, Golden NH, Feldman HM et al. Abnormal white matter properties in adolescent girls with anorexia nervosa. Neuroimage Clin 2015; 9: 648-659.

19. Yau WY, Bischoff-Grethe A, Theilmann RJ, et al.Alterations in white matter microstructure in women recovered from anorexianervosa. Int J Eat Disord 2013; 46: 701-708. 